\title{
Solution-Gated Graphene FET DNA sensor for using unique coplanar gate structure integrated in the microfluidic chip
}

\author{
Hyo Eun Kim, June Ho Lee, Ariadna Schuck and Yong-Sang Kim. * \\ School of Electronic and Electrical Engineering, Sungkyunkwan University, Suwon, Gyeonggi 16419, \\ Korea \\ E-mail: yongsang@skku.edu
}

\begin{abstract}
In this work, we have developed a solution-gated graphene field effect transistors (SGGFETs) for sensing single-stranded DNA by unique coplanar gate structure design. Coplanar electrodes and graphene active layer are all exposed outside the plane to integrate with microfluidic chip. Integrating the microfluidic into the FETs, a certain amount and path of the DNA solution can be controlled optionally by microfluidic channel. [1] The probe DNA $(10 \mu \mathrm{M})$ was injected through the microfluidic channel and immobilization directly onto non-treated chemical vapor deposition (CVD) graphene. The Dirac point was shifted to the positive direction $(130 \mathrm{mV} \sim 160 \mathrm{mV})$ after probe DNA immobilization. After the target DNA hybrid with probe DNA in different concentration $(10 \mu \mathrm{M} \sim 100 \mathrm{pM})$, Dirac point shift negative direction. However, Dirac point was not shift with non-complementary DNA. In recent study, positive shift of Dirac point after probe DNA immobilization indicates an p-doping effect, the increases of hole carrier concentration in graphene is dominated by positive charges. In contrast, hybridization of complementary DNA cause n-doping, that the nucleosides adhered to graphene play a key role in the negative shift of Dirac point. [2] The complementary DNA (100 pM) without any surface treatment on CVD graphene was confirmed as the limit of detection. For the observation of the hybridized target DNA, further interacted with green dye produced green fluorescence upon exposure.
\end{abstract}

Key words: coplanar gate, microfluidic chip, solution-gated graphene field effect transistors, CVD graphene, Dirac point 


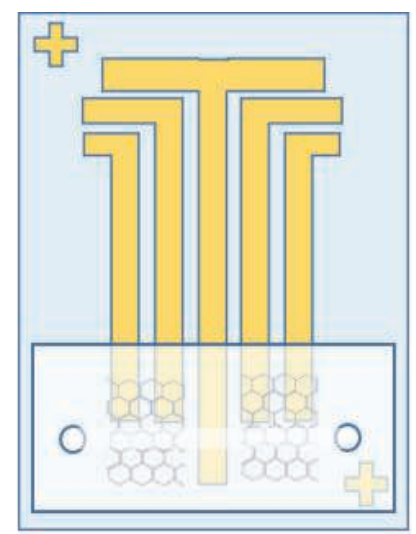

Fig. 1. schematic of the Microfluidic integrated SGGFETS

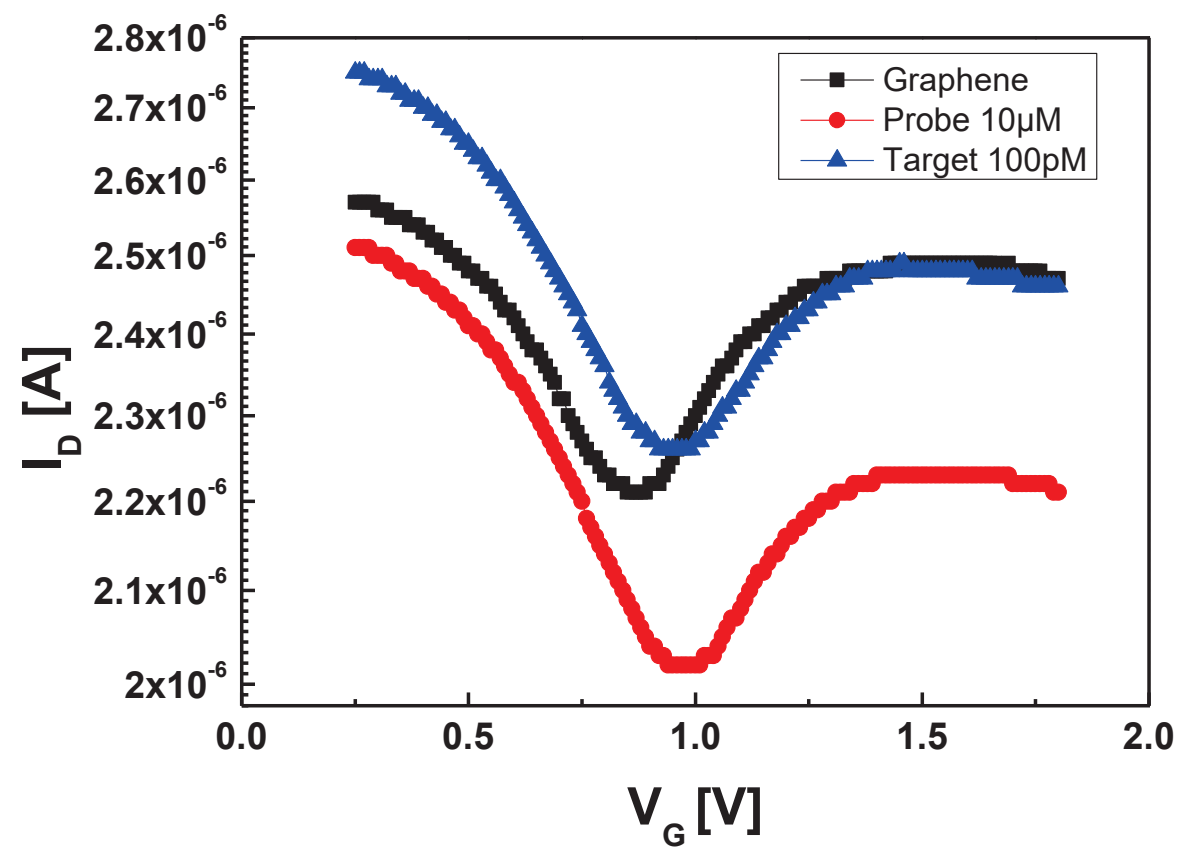

Fig. 2. Transfer characteristics of the probe DNA (10 $\mu \mathrm{M})$ immobilization and the complementary DNA (100pM) hybridization on CVD graphene

\section{References}

[1] D. Han, R. Chand, and Y-S Kim, Biosensors and Bioelectronics 93, 220-225 (2017); doi: 10.1016/j.bios.2016.08.115

[2] C-T. Lin, P.K. Loan, T-Y. Chen, K-K Liu, C-H Chen, K-H Wei, and $\mathrm{L}-\mathrm{J} \mathrm{Li}$, Advance Functional Materials 23, 2301-2307 (2013) doi: 10.1002/adfm.201202672 